\title{
RESULTADOS PRELIMINARES SOBRE LA VARIACION EN VULNERABILIDAD A LA CAVITACIÓN POR SEQUÍA EN CLONES DE Eucalyptus grandis Hill ex Maid.
}

\author{
Tesón, Natalia ${ }^{1}$; Fernández, María Elena² y Licata, Julián ${ }^{1}$
}

\section{RESUMEN}

El transporte de agua está ligado funcionalmente con la fijación de carbono en las plantas terrestres. El agua transportada dentro de la planta se encuentra en estado meta-estable debido a las presiones negativas de acuerdo a la Teoría de Cohesión-Tensión. Las curvas de vulnerabilidad a la cavitación, es decir, aquellas que relacionan las pérdidas de conductividad hidráulica (ks) del leño por ruptura de la columna de agua, con las tensiones dentro del mismo, dan valiosa información acerca de la capacidad de respuesta de distintas especies y genotipos al estrés hídrico.

El objetivo del estudio fue evaluar la variación en tolerancia al estrés hídrico de cuatro clones de Eucalyptus grandis (K, B, 2 y 4) del Programa de Mejoramiento Genético de INTA. Para ello, debió ajustarse una metodología adecuada para realizar las curvas de vulnerabilidad a la cavitación en árboles adultos. Se trabajó con el método de inyección de aire que relaciona la pérdida de conductividad hidráulica con la cavitación inducida por presiones positivas en una cámara de doble entrada. Los clones seleccionados presentan densidad de madera contrastante (alta/ baja) y distintas tasas de crecimiento.

El material evaluado se obtuvo de un ensayo clonal de 18 años de edad ubicado en Concordia, Entre Ríos, Argentina ( $31^{\circ} 22^{\prime} \mathrm{L} \mathrm{S}$; $58^{\circ} 07^{\prime} \mathrm{L}$ W; Altitud: $43 \mathrm{msnm}$ ) en un suelo arenoso profundo. Entre abril y mayo de 2012, mediante un hidroelevador se ascendió a las copas y se cortaron ramas de tres individuos por clon. Para la realización de las curvas de cavitación se utilizó la porción basal $(20 \mathrm{~cm})$ de las ramas secundarias. Se comparó con ANOVA los parámetros a y b de la curva de vulnerabilidad a la cavitación que describen el $P_{50} 0$ presión a la que se produce el $50 \%$ de pérdida de ks y la forma de la curva.

Los resultados obtenidos muestran diferencias significativas entre dos de los clones evaluados, siendo el más tolerante a la cavitación $\left(P_{50}=-1,85+/-0,19 \mathrm{MPa}\right)$ el clon 2 , que es uno de los clones de alta densidad de madera. El menos tolerante fue el clon $B\left(P_{50}=-1,11+/-\right.$ $0,11 \mathrm{MPa}$ ), caracterizado por ser de rápido crecimiento y baja densidad de madera. Estos resultados son congruentes con su respuesta post-sequía evaluada en crecimiento y flujo de savia (estudios previos), que mostró una mayor resiliencia en el clon 2 y una menor en el clon $\mathrm{B}$. El clon $\mathrm{K}$, de alta densidad, también presentó alta resiliencia post-sequía, pero no mostró ventajas claras en vulnerabilidad a la cavitación, aunque sí fue el de mayor ajuste osmótico en el tejido foliar.

Se concluye que la vulnerabilidad a la cavitación es variable entre clones, tendría un rol adaptativo importante en la resistencia a la sequía de $E$. grandis, pero existen mecanismos alternativos (y/o complementarios) en otros sistemas de órganos que deben también evaluarse con miras a la selección de genotipos más resistentes al estrés ambiental.

Palabras clave: Eucalyptus grandis, resistencia a sequía, cavitación.

\footnotetext{
${ }^{1}$ Instituto Nacional de Tecnología Agropecuaria (INTA), Estación Experimental Agropecuaria Concordia, Concordia, Provincia de Entre Ríos, Argentina. nteson@correo.inta.gov.ar

${ }^{2}$ Consejo Superior de Investigaciones Científicas y Técnicas (CONICET), INTA EEA Balcarce-Oficina Tandil, Tandil, Provinvia de Buenos Aires, Argentina.
} 


\section{SUMMARY}

Water transport is functionally linked to carbon fixation in terrestrial plants. Water inside plants is under meta-stable state due to negative pressure (tension) according to Cohesion-Tension Theory. Vulnerability to cavitation curves, i.e., those relating losses in xylem hydraulic conductivity (ks) due to water column breakage with tension inside the xylem, provide valuable information about different species and genotypes response capacity to environmental stress.

The objective of this study was to evaluate the variation in drought stress tolerance of four Eucalyptus grandis clones (K, B, 2 and 4 ) from the Genetic Improvement Program of INTA. For this purpose, methodological adjustments were needed in order to develop vulnerability to cavitation curves in adult trees. The air-injection method was applied, which relates ks losses with cavitation induced by positive pressures in a double-ended chamber. The selected clones present contrasting wood density (high, low) and different growth rates.

The studied material was obtained from a 18 years-old clonal trial located in Concordia, Entre Ríos, Argentina (S L: $31^{\circ} 22^{\prime}$ S; W L.: 58 07'; Altitude: 43 masl) in deep sandy soil. Crown access was possible with a hydraulic crane. Branch samples ( 3 individuals per clone) were taken between April and May 2012. Vulnerability to cavitation curves were developed in the basal portion (20 cm long) of secondary branches. Curves parameters ( $a$ and $b$ ) were compared between clones with ANOVA, describing the water potential at which $50 \%$ of ks is lost $\left(P_{50}\right)$ and the curve form.

Significant differences were observed between two studied clones. The most cavitation resistant clone $2\left(\mathrm{P}_{50}=-1.85+/-0.19 \mathrm{MPa}\right)$ was a high wood density clone. The least cavitation resistant clone $\mathrm{B}\left(\mathrm{P}_{50}=-1.11+/-0.11 \mathrm{MPa}\right)$ was characterized by its high growth rates and low wood density.

These results are consistent with post-drought responses evaluated through growth and sapflow measurements (previous studies) which demonstrated a higher resilience in clone 2 than in clone $\mathrm{B}$. High wood density clone $\mathrm{K}$ also presented a high resilience after a severe drought event, but it did not present clear advantages in terms of cavitation resistance. However, it presented the highest osmotic adjustment capacity of the studied clones.

In conclusion, vulnerability to cavitation is variable between clones and it would have an adaptive role in terms of $E$. grandis drought resistance. However, alternative (and/or complementary) mechanisms exist in other plant organs which have to be evaluated in order to select drought resistant genotypes.

Key words: Eucalyptus grandis, drought resistance, cavitation. 


\section{INTRODUCCIÓN}

En la provincia de Entre Ríos existen 144.732 ha con plantaciones forestales (MAGyP, 2012), el principal género botánico cultivado es Eucalyptus (71\% de la superficie) y casi la totalidad corresponde a la especie Eucalyptus grandis. Si bien bajo condiciones normales el comportamiento de la especie es muy bueno, alcanzando crecimientos de $56 \mathrm{~m}^{3} /$ ha/año con prácticas convencionales (Marcó 1998), la misma presenta importantes disminuciones de crecimiento ante condiciones de estrés hídrico y problemas de supervivencia y calidad de la madera ante ocurrencia de bajas temperaturas.

En la Estación Experimental Agropecuaria (EEA) Concordia del Instituto Nacional de Tecnología Agropecuaria (INTA) de Argentina se ha trabajado durante los últimos 30 años en el mejoramiento genético de la especie, evaluando principalmente volumen, forma y densidad de madera. Se han generado más de 300 clones puros de E. grandis y 150 clones híbridos en diversas etapas de evaluación, de los cuales 16 clones (10 de E. grandis y 3 híbridos de E. grandis $x$ E. camaldulensis y 3 de E. grandis $x$ E. tereticornis, están ya inscriptos en el Registro Nacional de Cultivares del INASE y liberados al mercado (Marco y Harrand, 2012).

La incidencia de estrés ambiental generada por eventos esporádicos en las actuales zonas forestadas, el incremento de los mismos en el tiempo debido al cambio climático global y la expansión de la superficie forestada a sitios más marginales debido a competencias con otros usos de la tierra, ha hecho que los programas de mejoramiento genético a nivel mundial reconozcan la necesidad de incorporar criterios de adaptabilidad a estrés dentro del proceso de selección. Es así como se requiere el aporte de disciplinas como la ecofisiología vegetal, que intenta desentrañar los procesos de respuesta de la planta ante distintas condiciones ambientales.

La capacidad de transporte de agua de una planta, cuantificada en la conductividad hidráulica (ks) de distintos órganos o la conductancia hidráulica $(\mathrm{K})$ de la planta entera, se relaciona funcionalmente con su capacidad de fijación de carbono (Hubbard et al., 2001; Santiago et al., 2004) y su crecimiento (Kondoh et al., 2006, Brodribb et al., 2005, Domec y Gartner, 2003). El transporte de agua desde las raíces a la atmósfera, de acuerdo a la teoría de Cohesión-Tensión, se desarrolla a través de un gradiente de potenciales hídricos negativos.

Bajo condiciones de estrés hídrico, ya sea por déficit de agua en el suelo y/o por alta demanda evaporativa de la atmósfera, se alcanzan condiciones adecuadas para que la columna de agua se rompa o cavite, es decir, el agua bajo tensión en el xilema cambia de estado, de líquido a gaseoso, generando embolismos en los conductos con la consecuente pérdida de conductividad hidráulica.

Cada especie y cada órgano dentro de una planta tiene una curva característica de vulnerabilidad a la cavitación (Tyree,1999) determinada por las características anatómicas de la madera que varían con la edad cambial, la posición dentro de la planta, la disponibilidad de recursos y el acervo genético de cada individuo. Es así que la estructura de los conductos encargados del transporte de agua en el xilema es de vital importancia, donde combinaciones determinadas de espesor de pared, diámetro de lúmen y cantidad, diámetro y ultra-estructura de las puntuaciones intervasculares otorgarían distintos grados de seguridad en el transporte de agua (Zimmermann, 1983, Hacke et al., 2001). Es por ello que este carácter posee un alto valor adaptativo en numerosas especies leñosas (Pockman \& Sperry, 2000; Hacke et al., 2000).

A una escala de menor detalle, ciertas propiedades de la madera emergentes de las características anatómicas mencionadas, como su densidad, se relacionan con las propiedades hidráulicas del xilema (Hacke y Sperry, 2001, Sperry et al., 2008; Pittermann et al., 2006). La densidad, variable de gran valor tecnológico para la madera, es el resultado de la proporción de lúmenes y paredes celulares. La relación entre densidad de madera y resistencia a la cavitación por sequía no sería, sin embargo, una relación causal sino una correlación. Se ha postulado que 
los conductos capaces de resistir altas tensiones sin cavitar deben desarrollar paredes celulares gruesas de manera de evitar la implosión o colapso del conducto (Hacke et al., 2001, Jacobsen et al., 2005). De esta manera, maderas más resistentes a la cavitación deberían tener una alta proporción de pared celular en relación con los lúmenes, resultando en altas densidades de madera. Es por ello que resulta interesante conocer las correlaciones entre densidad de madera y seguridad del sistema de conducción, de manera de usar la primera como método indirecto de selección de la segunda.

Si bien existe innumerable cantidad de trabajos que han cuantificado la vulnerabilidad a la cavitación del xilema en distintas especies latifoliadas y coníferas, son muy pocos los estudios de este tipo desarrollados en especies del género Eucalyptus. Este género posee una madera compleja, conformada por distintos tipos celulares (vasos, fibras, fibrotraqueidas, traqueidas vasicéntricas), cuyo valor funcional no ha sido aún dilucidado. Es por ello que se ha iniciado una serie de estudios que relacionan la anatomía y función de la madera de distintas especies de este género implantadas en Argentina, así como sus implicancias con respecto a posibles compromisos con el crecimiento. En este sentido, en términos generales, se plantea que existe un compromiso entre capacidad de conducción de agua (alta productividad) y alta resistencia a sequía-frío debido a que ambos procesos se relacionan inversamente con el tamaño de los elementos del sistema de conducción (Kondoh et al., 2006; Domec et al., 2006; Sperry et al., 2008). La información expuesta en este artículo es parte de este estudio, presentándose los primeros resultados sobre vulnerabilidad a la cavitación de cuatro clones de E. grandis del Programa de Mejoramiento Genético de INTA, seleccionados en este estudio por poseer densidad de madera y tasas de crecimiento contrastantes.

Los resultados de seguridad del sistema de conducción se discuten posteriormente a la luz de los de crecimiento y uso de agua de los mismos clones bajo condiciones de campo, caracterizadas por un evento de sequía severo (estudios previos) de manera de comenzar a descifrar el posible rol adaptativo de los caracteres funcionales de la madera encontrados.

\section{MATERIALES Y METODOS}

El material evaluado se obtuvo de un ensayo clonal de productividad, instalado en el año 1994, en Concordia, Entre Ríos, Argentina (3122' LS; 58 $07^{\circ}$ LW y Altitud 43 msnm) en un suelo arenoso profundo. Los clones seleccionados para este estudio presentan densidad de madera contrastante (alta/ baja) y distintas tasas de crecimiento (Cuadro $\mathrm{N}^{\circ} 1$ ).

Los clones 2 y $\mathrm{K}$ presentan relativamente alta densidad de madera, mientras que los clones 4 y B son de baja densidad. Los clones 4 y K son los más contrastantes en crecimiento, presentando diferencias significativas entre los mismos. Distintos individuos de estos clones fueron estudiados entre 2007 y la actualidad, analizándose su crecimiento en altura y diámetro a la altura del pecho, densidad de flujo de savia, capacidad de ajuste osmótico del tejido foliar, anatomía de la madera, etc. Algunos resultados han sido presentados en Tesón et al. (2011) y otros están siendo volcados en un manuscrito en preparación.

Entre abril y mayo de 2012, mediante un hidroelevador se ascendió a las copas y se cortaron ramas de tres individuos por clon. Se realizaron las curvas de vulnerabilidad a la cavitación en la porción basal $(20 \mathrm{~cm})$ de las ramas secundarias, utilizando el método de inyección de aire que relaciona la pérdida de conductividad con la cavitación inducida por presiones positivas en una cámara de doble entrada.

La metodología general aplicada es la usual para esta técnica (descripta por ejemplo en Gyenge y Dalla Salda, 2010), aunque debió desarrollarse el protocolo particular para la especie (no existían antecedentes), ensayándose distintos tiempos de aplicación de presión, de estabilización post-presión y de medición de ks. 
Se trazaron curvas de vulnerabilidad a la cavitación relacionando las pérdidas de conductividad (\% PLC) con la presión positiva aplicada (MPa), ajustándoseles el modelo de Pammenter y Vander Willigen (1998):

$$
\mathrm{PLC}=100 /\left[1+\exp \left(a^{*}(\psi-b)\right)\right]
$$

Donde: PLC es el porcentaje de pérdida de ks

a es el grado de curvatura de la curva

b es el potencial al que se alcanza $50 \%$ de pérdida de ks $\left(\mathrm{P}_{50}\right)$ $\psi$ es la presión positiva ejercida en la cámara de doble entrada (equivalente al potencial hídrico del xilema al cual se producen los distintos PLC).

A partir de los parámetros a y b de las curvas se estimaron también los parámetros $\mathrm{Pe}$ y Pmax, que representan el punto de entrada de aire $(P L C=12)$, o potencial hídrico a partir del cual la ks decrece rápidamente por aumento de la cavitación, y el punto de máxima cavitación ( $\mathrm{PLC}=88$ ), según las fórmulas:

$$
\begin{aligned}
& P e=(2 / a)+b \\
& P \max =(-2 / a)+b
\end{aligned}
$$

Los modelos de regresión se ajustaron mediante el Programa Informático Table Curve 2D (Jandel Scientific, EEUU). Los parámetros de las curvas se compararon mediante ANOVA y posteriores tests de Tukey.

\section{Cuadro $\mathrm{N}^{\circ} 1$

\begin{tabular}{|c|c|c|}
\hline Clon & 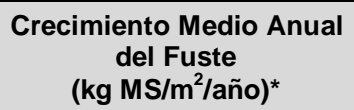 & $\begin{array}{c}\text { Densidad de Madera } \\
\qquad\left(\mathrm{kg} / \mathrm{m}^{3}\right)^{\star}\end{array}$ \\
\hline 2 & $1,29(0.69) \mathrm{ac}$ & $444,2(23.5) \mathrm{a}$ \\
\hline 4 & $1,14(0.45) b c$ & $401,1(14.7) b$ \\
\hline $\mathrm{K}$ & $1,42(0.26)$ ac & $439,5(10.5) \mathrm{a}$ \\
\hline$B$ & $1.72(0.53) \mathrm{a}$ & $397.2(13.2) b$ \\
\hline
\end{tabular} \\ CRECIMIENTO ANUAL EN BIOMASA DE FUSTE Y DENSIDAD DE MADERA MEDIDOS EN CUATRO CLONES DE Eucalyptus grandis}

\section{RESULTADOS}

El protocolo seleccionado para la confección de las curvas definitivas consistió en la aplicación de 5 minutos de cada uno de los niveles de presión (entre 0 y $3 \mathrm{MPa}$; cada $0,25 \mathrm{MPa}$ en los primeros puntos y cada 0,5 MPa a partir de 1,5 MPa), seguidos de 5 minutos de estabilización a presión atmosférica, luego de los cuales se registró el pasaje de agua por la rama durante 10 minutos.

Los cuatro clones presentaron curvas con una forma general similar (Figura $\mathrm{N}^{\circ} 1$ ), caracterizada por una caída gradual de la ks, con un promedio general de $P_{50}$ de 1,5 (DS: 0,32 ) $\mathrm{MPa}$. En general, los ajustes de los modelos fueron muy buenos, con un promedio de $\mathrm{R}^{2}$ ajustado para todas las curvas ( 1 curva por individuo) de 0,88 (mínimo $R^{2}: 0,78$; máximo $R^{2}: 0,99$ ). 


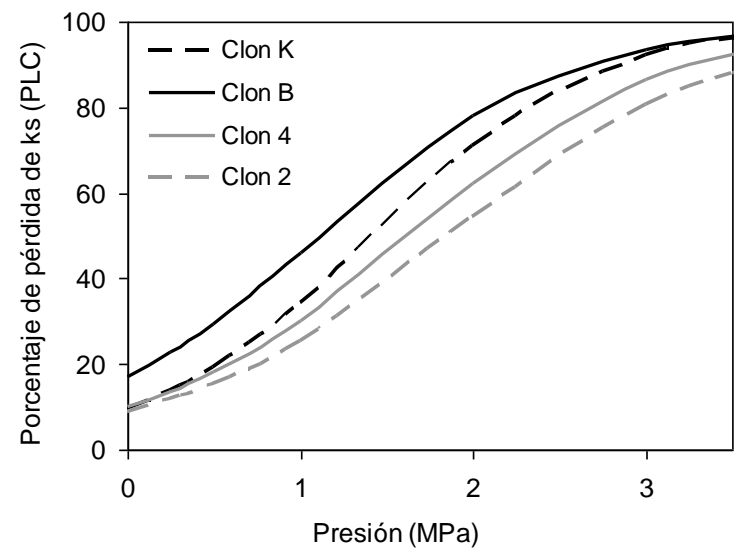

Se grafican los modelos ajustados a los datos según la formula de Pammenter y Vander Willigen, 1998.

\section{FIGURA $N^{\circ} 1$ \\ CURVAS DE VULNERABILIDAD A LA CAVITACIÓN DE LOS CUATRO CLONES \\ DE Eucalyptus grandis}

El único parámetro de la curva que mostró diferencias significativas entre algunos clones fue el $\mathrm{P}_{50}$, observándose diferencias significativas entre el clon 2 y el $\mathrm{B}$ (Figura $\mathrm{N}^{\circ} 2$ ). Tanto el parámetro de forma de la curva (promedio general DS: $-1,65(0,46)$ ), como el Pe (promedio general (DS): 0,26 $(0,29) \mathrm{MPa})$ y el Pmax (promedio general (DS): 2,80 $(0,58) \mathrm{MPa})$ no mostraron diferencias significativas entre clones, aunque se observó la tendencia a que el clon B presentara valores de entrada de aire posteriores (a mayores tensiones) que los otros clones.

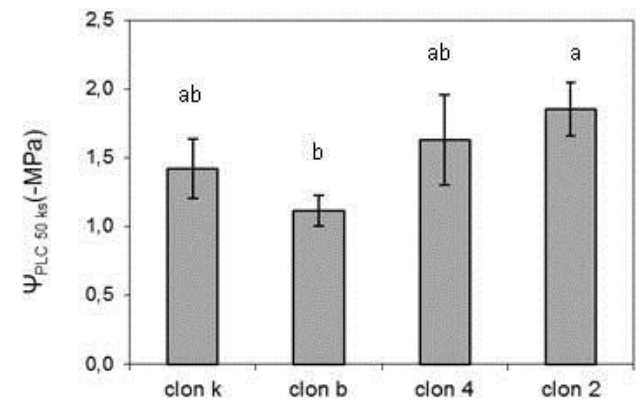

Las barras de error representan un desvío estándar. Letras diferentes indican diferencias significativas $(p<0,05)$ entre los clones.

FIGURA $\mathbf{N}^{\circ} 2$

VALORES MEDIOS DE PRESIÓN APLICADA QUE CAUSARON LA DISMINUCIÓN DEL 50\% DE LA CONDUCTIVIDAD HIDRÁULICA ( $\mathrm{P}_{50}$, CORRESPONDIENTE A PLC $\left.=50 \%\right)$. 


\section{DISCUSION}

El grado de diferenciación en $\mathrm{P}_{50}$ observado entre los distintos clones es interesante (diferencia máxima media de $0,75 \mathrm{MPa}$ ), aunque cabe mencionar que estos resultados deben ser tomados como preliminares debido a que el tamaño muestral utilizado fue bajo (debido a la alta laboriosidad que implica la confección de estas curvas). Sin embargo, sugieren que existe variabilidad en la vulnerabilidad a la cavitación de la madera de ramas entre los clones de Eucalyptus grandis analizados.

Un antecedente de curvas de cavitación en E. grandis (un único clon sudafricano) mostró un $\mathrm{P}_{50}$ de $-1,31 \mathrm{MPa}$, con una diferenciación máxima (y significativa) de 0,46 MPa con los otros clones evaluados, que eran híbridos de E. grandis con E. camaldulensis (Vander Willigen \& Pammenter, 1998). Un estudio en estacas enraizadas de cuatro clones de E. globulus mostró un grado de diferenciación en el $\mathrm{P}_{50}$ de tan sólo 0,18 MPa entre los mismos (Pita et al., 2003).

El parámetro que describe la forma de la curva fue menor en el presente trabajo $(1,65)$ que en el mencionado anteriormente de $E$. grandis (2,25; Vander Willigen \& Pammenter, 1998), lo que indica una caída menos abrupta de la ks a medida que aumenta la presión (o tensión, según el método de confección de la curva) en los clones argentinos.

El clon de E. grandis que mostró la mayor resistencia a la cavitación (clon 2) fue el de mayor densidad de la madera (Cuadro $N^{\circ} 1$ ), mientras que el más vulnerable (clon $\mathrm{B}$ ) fue el de menor densidad. Debe considerarse, sin embargo, que la densidad de la madera es una variable evaluada en el fuste, mientras que la vulnerabilidad a la cavitación fue ensayada en ramas. Si bien es de esperarse que exista una coordinación general entre los distintos órganos de la planta, esto no necesariamente implica que los mismos patrones de vulnerabilidad a la cavitación sean encontrados en el fuste. Incluso, muchas veces existe el fenómeno de "vulnerabilidad segmentada" (sensu Zimmermann, 1983) por el cual los órganos distales de una planta son mucho más vulnerables que otros, de manera de que ante un evento de sequía severa actúan como "fusible" del sistema hidráulico, cavitando prontamente y disminuyendo la superficie transpirante (por absición de las hojas). Un mecanismo de este tipo ha sido descripto para juveniles (estacas enraizadas) de E. globulus (Pita et al, 2003). De todas maneras, resultados preliminares no publicados (Monteoliva et al, UNLP, en el marco del mismo proyecto de investigación) muestran que existe una correlación en los caracteres microscópicos de la madera del fuste y de las ramas dentro de cada clon.

Que exista variación en vulnerabilidad a la cavitación de la madera entre distintos genotipos no implica necesariamente que este carácter pueda tener un rol funcional significativo para la resistencia al estrés hídrico en $E$. grandis. En este sentido, si bien se sabe que es un carácter determinante en la resistencia al estrés ambiental en gran cantidad de especies leñosas, son muy pocos los antecedentes que han evaluado el rol adaptativo de la vulnerabilidad a la cavitación en el género Eucalyptus. Uno de ellos es el trabajo de Vander Willigen \& Pammenter (1998) mencionado en el párrafo anterior. En él se menciona que el clon de E. grandis evaluado, que fue el menos resistente a la cavitación en comparación con híbridos interespecíficos, sufrió un $90 \%$ de mortalidad aérea en una sequía severa ocurrida en el año 1992 en Sudáfrica. En el caso de E. globulus, su curva de vulnerabilidad a la cavitación lo ubica entre las especies más vulnerables, sufriendo pérdidas importantes de conductividad durante su temporada de crecimiento (Pita et al., 2005). Estos autores han sugerido que esta especie posee alta productividad a expensas de estar siempre al borde del embolismo catastrófico, lo que seguramente resulta muy riesgoso si las condiciones de estrés hídrico aumentan.

En el caso del presente estudio, las curvas de vulnerabilidad a la cavitación pueden ser analizadas a la luz de información de la respuesta de los cuatro clones a un evento de sequía severa ocurrida en la primavera-verano 2008-2009 (Tesón et al, 2011). Esta respuesta fue evaluada en crecimiento antes, durante y post-evento de sequía, así como la densidad de flujo de savia de los árboles, que puede ser utilizada como proxy de su capacidad de fijación de carbono. 
Los clones 2 y $\mathrm{K}$ (ambos de alta densidad de madera) fueron los que se destacaron por su alta resiliencia post-sequía (rápida recuperación del crecimiento), mientras que los clones B y 4 (de baja densidad de madera) presentaron una recuperación del crecimiento y transporte de agua más lentos (menor resiliencia). La alta resiliencia post-sequía de los dos clones de alta densidad podría ser explicada en parte por una mayor resistencia a la cavitación (observada solo en el clon 2). El clon K, si bien no mostró ventajas particulares en cuanto a su vulnerabilidad a la cavitación (fue intermedia entre la de los otros clones), sí presentó una alta capacidad de ajuste osmótico en su tejido foliar, carácter que ha sido descripto como de importancia adaptativa para el género (Guarnaschelli et al., 2003). Durante el evento de sequía, sin embargo, el clon $\mathrm{K}$ presentó claras ventajas por sobre el 2 (menor caída del crecimiento y flujo de agua), lo que sugiere que el ajuste osmótico foliar podría tener un rol crucial en el mantenimiento de la fijación de $\mathrm{C}$ durante un evento de sequía. El clon $B$, de mayor vulnerabilidad a la cavitación, se destaca también por una anatomía de la madera del fuste bastante diferenciada con respecto a la de los otros clones (Tesón et al., 2011; Monteoliva et al, datos no publicados aún), altas tasas de crecimiento cuando la disponibilidad de agua es alta, una alta sensibilidad a la sequía y una baja resiliencia post-sequía. Los resultados de este trabajo sugieren que la cavitación del sistema de conducción, junto con una baja capacidad de ajuste osmótico, pueden ser responsables importantes de esta baja resistencia al déficit hídrico.

Comparando las curvas de cavitación para cada clon con los valores de potencial hídrico de los mismos observados en distintos momentos de la temporada de crecimiento (datos no publicados, disponibles en el Informe Final del Proyecto INTA 2213), puede estimarse que en condiciones normales, cuando los clones alcanzan valores de potencial hídrico de alrededor de -1,5 $\mathrm{MPa}$, todos estarían sufriendo pérdidas de aproximadamente el $50 \%$ de la ks de las ramas. En condiciones de sequía extrema, en las que el clon $\mathrm{B}$ se diferenció de los otros por alcanzar potenciales al mediodía de -3 a $-3,5 \mathrm{MPa}$, mientras que los otros alcanzaron valores de $-2,5$ a $-2,8$ $\mathrm{MPa}$, las curvas indican que el clon B tendría una pérdida total de la ks coincidente con la gran absición foliar que se observó en el mismo (observación personal de los autores), mientras que los clones 4 y K perderían poco más del $90 \%$ de ks, y el clon 2 , más resistente, alrededor del $80 \%$ de la ks.

Curvas de cavitación realizadas un mes después de las presentadas en el presente artículo muestran que existe una fuerte variación temporal en este carácter, posiblemente ligada a la producción de nueva madera con características anatómicas diferentes en muy corto plazo.

Actualmente se están llevando a cabo estudios que complementan al presente de manera de describir mejor el rol funcional y su variación estacional de la vulnerabilidad a la cavitación en E. grandis.

\section{CONCLUSIONES}

En base a estos primeros resultados se concluye que la vulnerabilidad a la cavitación del xilema podría tener un rol adaptativo importante en $E$. grandis, especialmente explicando la baja resistencia a la sequía de algunos genotipos (con alta vulnerabilidad a la cavitación, como el clon B). Si bien una alta resistencia a la cavitación se vio acompañada con una alta resiliencia postsequía (como en el clon 2), esta no alcanza por sí misma para garantizar un buen desempeño durante un evento de sequía (baja sensibilidad).

Caracteres adicionales, como la capacidad de ajuste osmótico, deberían también ser, siempre que pueda medírselos de manera relativamente expedita, contemplados a la hora de seleccionar genotipos con baja sensibilidad a la sequía y alta resiliencia post-sequía (como el clon $\mathrm{K})$.

La densidad de la madera parece ser un carácter asociado a la adaptabilidad a la sequía en $E$. grandis, sin tener necesariamente compromisos con la productividad (como en el caso del 
clon K), lo que abre un panorama interesante para utilizarla como método de screening para adaptabilidad (más allá de su valor tecnológico) en Programas de Mejoramiento Genético, en combinación con caracteres foliares (área foliar específica, tamaño medio de la hoja, ajuste osmótico) y máxima ks.

\section{RECONOCIMIENTOS}

Este estudio ha sido financiado por el subsidio PNFOR 042131 de INTA (Argentina) Bases Ecofisiológicas para la Producción de Madera en Cantidad y Calidad bajo Distintas Condiciones Ambientales.

\section{REFERENCIAS}

Brodribb, T. J.; Holdbrook, N. M. and Hill, R. S., 2005. Seedling growth in conifers and angiosperms: Impacts of constrasting xylem structure. Aust J Bot. 53: 749-755.

Domec, J. C. y Gartner, B. L., 2003. Relationship between growth rates and xylem hydraulic characteristics in young, mature and old-growth Ponderosa Pine trees. Plant Cell Environ 26: 471-483.

Domec, J. C.; Lachenbruch, B. and Meizner, F. C., 2006. Bordered pit structure and function determine spatial Patterns of air-seeding thresholds in xylem of Douglas-Fir (Pseudotsuga menziesii; Pinaceae) trees. Am J Bot. 93: 1588-1600.

Guarnaschelli, A. B.; Lemcoff, J. H.; Prystupa, P. y Basci, S. O., 2003. Responses to drought preconditioning in Eucalyptus globulus Labill. provenances. Trees 17: 501-509.

Gyenge, J. E. y Dalla Salda, G., 2010. Cap. 5: Curvas de vulnerabilidad a la cavitación. En: Fernández, M. E. \& Gyenge, J. E. Eds. En: Técnicas de medición en ecofisiología vegetal: conceptos y procedimientos. Ediciones INTA Ediciones INTA, Buenos Aires, Argentina. ISBN 978-987-1623-76-1, pág. 69-78.

Hacke, U.; Sperry, J.; Pockman, W.; Davis, S. and McCulloh, K., 2001. Trends in wood density and structure are linked to prevention of xylem implosion by negative pressure. Oecologia 126: 457-461.

Hacke, U.G.; Sperry, J. S. y Pittermann, J., 2000. Drought experience and cavitation resistance in six shrubs from the Great Basin, Utah. Basic and Applied Ecology 1: 31-41.

Hacke, U. G. and Sperry, J. S., 2001. Functional and ecological xylem anatomy. Perspectives in Plant Ecol Evol Syst. 4: 97-115.

Hubbard, R. M.; Ryan, M. G.; Stiller, V. and Sperry, J. S., 2001. Stomatal conductance and photosynthesis vary linearly with plant hydraulic conductance in Ponderosa Pine. Plant Cell Environ. 24: 113-121.

Jacobsen, A. L.; Ewers, F. W.; Pratt, R. B.; Paddock, W. A. and Davis, S. D., 2005. Do Xylem Fibers Affect Vessel Cavitation Resistance? Plant Physiol. 139: 546-556.

Kondoh, S.; Yahata, H.; Nakashizuka, T. and Kondoh, M., 2006. Interspecific variation in vessel size, growth and drought tolerance of broad-leaved trees in semi-arid regions of Kenya. Tree Physiol. 26: 899-904.

Marcó, M. A., 1988. Crecimiento del Eucalyptus grandis en diferentes suelos de Concordia. Resultados a los 10,5 años. VI Congreso Forestal Argentino. Santiago del Estero, Argentina. Tomo II: 510-511.

Marcó, M. A. y Harrand, L., 2012. El programa de mejora de Eucaliptos del INTA en la Mesopotamia Argentina. Resúmenes Jornadas de Actualización Técnica Mejoramiento Genético de Pinos y Eucaliptos Subtropicales. EEA Concordia. Ediciones INTA: 44-48.

MAGyP, 2012. Ministerio de Agricultura Ganadería y Pesca. Presidencia de la Nación. Elaboración de un mapa de plantaciones forestales (MPF) de la República Argentina de actualización permanente. Área SIG e Inventario Forestal. Dirección de Producción Forestal. En: http://www.minagri.gob.ar/new/00/forestacion/inventario/mapa\%20de\%20planta.htmIMAGyP. (Consulta 22 de octubre de 2012) 
Pammenter, N. W. and Vander Willigen, C., 1998. A mathematical and statistical analysis of the curves illustrating vulnerability of xylem to cavitation. Tree Physiol 18: 589-593

Pita, P.; Gascó, A. and Pardos, J. A., 2003. Xylem cavitation, leaf growth and leaf water potential in Eucalyptus globulus clones under well-watered and drought conditions. Funct Plant Biol. 30: 891-899.

Pita, P.; Cañas, I.; Soria, F.; Ruiz, F. and Toval, G., 2005. Use of physiological traits in tree breeding for improved yield in drought-prone environments. The case of Eucalyptus globulus. Invest Agrar: Sist Recur For 14(3), 383-393

Pittermann, J.; Sperry, J. S.; Hacke, U. G.; Wheeler, J. K. and Sikkema, E. H., 2006. Inter-tracheid pitting and the hydraulic efficiency of conifer wood: the role of tracheid allometry and cavitation protection. Am. J. Bot 93 : 1265-1273.

Pockman, W.T. and Sperry, J. S., 2000. Vulnerability to cavitation and the distribution of Sonoran Desert vegetation. Am J Bot 87: 1287-1299.

Santiago, L. S.; Goldstein, G.; Meinzer, F. C.; Fisher, J. B.; Machado, K.; Woodruff, D. and Jones, T., 2004. Leaf hotosynthetic traits scale with hydraulic conductivity and wood density in Panamanian forest canopy trees. Oecologia 140: 543-550.

Sperry, J. S.; Meinzer, J. C. and McCulloh, K, A., 2008. Safety and efficiency conflicts in hydraulic architecture: scaling from tissues to trees. Plant Cell Environ. 31: 632-645.

Tesón, N.; Monteoliva, S.; Licata, J. A. y Fernández, M. E., 2011. Ecophysiological processes and wood anatomy related to growth and drought resistance in genotypes of Eucalyptus grandis. IUFRO 2011 Eucalyptus: Improvement and culture of eucalypts. Porto Seguro, Brasil, 14-18 de Noviembre de 2011, 3 pp.

Tyree, M. T., 1999. Water relations and hydraulic architecture. In: Handbook of functional plant ecology. Pugnire, F.I. \& F. Valladares. Ed. Marcel Dekker, New York. pp. 221-268.

Vander Willigen, C. and Pammenter, N. W., 1998. Relationship between growth and xylem hydraulic characteristics of clones of Eucalyptus spp. at contrasting sites. Tree Physiol. 18: 595-600.

Zimmermann, M. H., 1983. Xylem structure and the ascent of sap. Springer Verlag, Berlín. 